11,12

\title{
Плотность состояний и структура основного состояния модели Изинга на решетке Кагоме с учетом взаимодействия ближайших и следующих соседей
}

\author{
(C) М.А. Магомедов ${ }^{1,2}$, А.К. Муртазаев ${ }^{1,2}$ \\ ${ }^{1}$ Институт ффизики им. Х.И. Амирханова ДНЦ РАН, \\ Махачкала, Россия \\ ${ }^{2}$ Дагестанский государственный университет, \\ Махачкала, Россия \\ E-mail: magomedov_ma@mail.ru
}

Методом Монте-Карло изучены фазовые переходы и термодинамические свойства в двумерной антиферромагнитной модели Изинга на решетке Кагоме с учетом взаимодействия как ближайших, так и следующих соседей. На основе гистограммного метода анализа данных показано, что в исследуемой модели наблюдается фазовый переход второго рода. Обнаружено аномальное поведение в температурной зависимости термодинамических параметров.

DOI: $10.21883 /$ FTT.2018.06.45996.14M

\section{1. Введение}

В последнее время исследованию фазовых переходов (ФП), критического поведения и термодинамических свойств (ТС) низкоразмерных систем уделяется значительное внимание. Интерес к таким системам стимулируется большим количеством экспериментальных работ на квазиодномерных и квазидвумерных магнитных системах [1-11]. В настоящей работе нами проведены высокоточные исследования двумерной модели Изинга на решетке Кагоме с учетом взаимодействия вторых ближайших соседей. Модель Изинга на решетке Кагоме может быть применена для описания ряда двумерных материалов, в которых наблюдаются необычные температурные зависимости различных термодинамических параметров. Спиновые системы, имеющие решетку Кагоме, вследствие особой геометрии часто оказываются сильно фрустрированными. В таких системах основное состояние оказывается сильно вырожденным и часто не происходит упорядочения даже при самых низких температурах, вплоть до абсолютного нуля $[1,2]$.

В спиновых системах на решетке Кагоме при учете обменных взаимодействий только между ближайшими соседями ФП в магнитоупорядоченное состояние не реализуется ни при каких конечных значениях температуры. Учет обменных взаимодействий следующих ближайших соседей частично снимает вырождение и может привести к возникновению ФП при отличных от нуля температурах [3]. Тем не менее, поскольку эффекты фрустраций все еще имеют место, процесс упорядочения и стабилизации структур, в отличие от нефрустрированных систем, замедлен [4].

Влияние фрустраций, возникающих при конкурирующих взаимодействиях, обусловленных геометрией решетки, на ФП, термодинамические, магнитные и критические свойства спиновых систем исследовано в рабо- тах [5-8]. Авторами исследованы явления возникновения и исчезновения фрустраций в зависимости от величины внешнего магнитного поля, знаков констант обменного взаимодействия ближайших и следующих соседей $J_{1}$ и $J_{2}$ и величины их отношения $r=J_{2} / J_{1}$.

Несмотря на достигнутые успехи, на сегодняшний день все еще остаются открытыми некоторые вопросы, касающиеся ФП, критических, термодинамических и магнитных свойств спиновых систем с фрустрациями. Следует иметь в виду, что при исследовании таких систем очень важно знать, что в системе может существовать огромное количество состояний с низкой энергией, близкой к энергии основного состояния. Эти состояния благодаря своей большой энтропии могут вносить конечный вклад в термодинамику даже в пределе низких температур.

Модель Изинга на решетке Кагоме является одной из интенсивно исследуемых в последние годы фрустрированных моделей [9]. Данная модель является примером геометрически фрустрированной системы. В этой модели с взаимодействиями ближайших соседей в основном состоянии энтропия, приходящаяся на один спин, отлична от нуля [10]. Поэтому в данной системе ФП отсутствует при любой конечной температуре. Это связано с тем, что спиновое упорядочение сильно подавлено изза эффектов фрустраций. Однако учет взаимодействий следующих ближайших соседей стабилизирует спиновое состояние, и в системе возможно упорядочение и наблюдается ФП $[4,9]$.

В связи с этим, в настоящем исследовании нами рассматривается двумерная антиферромагнитная модель Изинга на решетке Кагоме с учетом взаимодействий следующих ближайших соседей. Кроме того, интерес к этой модели обусловлен тем, что она может быть использована для описания реальных материалов и соединений [12-17]. 
Поэтому для лучшего понимания термодинамического поведения систем с конкурирующими взаимодействиями существует необходимость проведения дополнительных более точных исследований антиферромагнитной модели Изинга на решетке Кагоме с учетом взаимодействия следующих ближайших соседей с использованием дополнительных современных идей и методов.

Исследование этой модели позволит получить ответ на ряд вопросов, связанных с характером магнитного упорядочения при низких температурах и природой ФП.

\section{2. Модель и метод исследования}

Модель Изинга на решетке Кагоме с учетом взаимодействий как первых так и вторых ближайших соседей описывается следующим гамильтонианом [18]:

$$
H=J_{1} \sum_{\langle i, j\rangle}\left(S_{i} \cdot S_{j}\right)+\sum_{\langle i, l\rangle}\left(S_{i} \cdot S_{l}\right),
$$

где $S_{i, j, l}= \pm 1-$ изинговский спин. Первый член в формуле (1) учитывает антиферромагнитное обменное взаимодействие ближайших соседей с константой $J_{1}>0$, а второй - ферромагнитное взаимодействие следующих соседей с константой $J_{2}<0$. В настоящей работе нами рассмотрен случай $J_{1}=1, J_{2}=-1$.

Строго и последовательно на основе микроскопических гамильтонианов такие системы могут быть изучены методами Монте-Карло (МК) [19-25]. В последнее время разработано много новых вариантов метода МК. Одним из наиболее эффективных для исследования подобных систем, особенно в низкотемпературной области, является алгоритм Ванга-Ландау [26,27]. Данный алгоритм является реализацией метода энтропийного моделирования и позволяет рассчитать функцию плотности состояний системы. Алгоритм Ванга-Ландау основан на том, что совершая случайное блуждание в пространстве энергий с вероятностями, обратно пропорциональными плотности состояний $g(E)$, мы получаем равномерное распределение по энергиям. Подобрав вероятности переходов так, чтобы посещение всех энергетических состояний происходило с одинаковой частотой, можно получить изначально неизвестную плотность состояний $g(E)$, зная которую можно вычислить значения необходимых термодинамических параметров при любой температуре. Так как плотность состояний $g(E)$ очень быстро растет с увеличением размеров исследуемых систем, для удобства хранения и обработки больших чисел пользуются величиной $\ln g(E)$.

Алгоритм Ванга-Ландау в настоящей работе был использован в следующем виде.

Задается произвольная начальная конфигурация спинов. Стартовые значения плотности состояний $g(E)=1$, гистограммы распределений по энергиям $H(E)=0$, стартовый модификационный фактор $f=f_{0}=e^{1}$ $\approx 2.71828$. Многократно совершаем шаги в фазовом пространстве, пока не получим относительно плоскую гистограмму $H(E)$ (т.е. пока не будут посещены примерно одинаковое количество раз все возможные энергетические состояния системы). При этом вероятность перехода из состояния с энергией $E_{1}$ в состояние с энергией $E_{2}$ определяется по формуле $p=g\left(E_{1}\right) / g\left(E_{2}\right)$. Если переход в состояние с энергией $E_{2}$ состоялся, то $g\left(E_{2}\right)$ присваивается значение $f \times g\left(E_{2}\right)$ и $H\left(E_{2}\right)$ увеличивается на единицу, иначе $g\left(E_{2}\right)$ присваивается значение $f \times g\left(E_{1}\right)$ и $H\left(E_{1}\right)$ увеличивается на единицу. Если гистограмма стала „Плоской“, то обнуляем гистограмму $H(E) \rightarrow 0$, уменьшаем модификационный фактор $f \rightarrow \sqrt{f}$ и продолжаем снова, пока $f \geq f_{\min }$. В нашем случае $f_{\min }=1.0000000001$.

В стандартную методику алгоритма мы добавили следующую процедуру, которая в итоге позволяет определить магнитную структуру основного состояния системы. Каждый раз при достижении энергетического минимума нами проводился анализ магнитной структуры основного состояния. При этом проводилось сравнение данной конфигурации с полученными ранее, и при обнаружении новой уникальной конфигурации производилось ее сохранение в специальной базе данных и вывод в текстовый файл всей необходимой информации (координаты каждого спина, его значение, внутренняя энергия системы и т.д.) а также запись в графический файл. Данная процедура позволяет избежать дублирования многократно встречающихся состояний с одинаковой магнитной структурой. Таким образом, если основное состояние не вырождено, то в базе данных в конце процесса моделирования окажется некоторое ограниченное количество конфигураций (для ферромагнитной модели Изинга, к примеру, с учетом симметрии относительно одновременного отражения всех спинов на решетке в базе окажутся две магнитные конфигурации). Для фрустрированных систем количество таких конфигураций будет бесконечным, при этом для экономии дискового пространства в памяти сохраняется не более 100 конфигураций. Более подробно алгоритм Ванга-Ландау изложен в работах [8,28-34].

Таким образом, выполнив последовательность описанных выше действий, можно рассчитать плотность состояний системы $g(E)$, зная которую, достаточно легко рассчитать значения любых термодинамических параметров при любой температуре. В частности, внутреннюю энергию $U$, свободную энергию $F$, энтропию $S$ можно вычислить, используя следующие выражения:

$$
\begin{gathered}
U(T)=\frac{\sum_{E} E g(E) e^{-E / k_{B} T}}{\sum_{E} g(E) e^{-E / k_{B} T}} \equiv\langle E\rangle_{T}, \\
F(T)=-k_{B} T \ln \left(\sum_{E} g(E) e^{-E / k_{B} T}\right), \\
S(T)=\frac{U(T)-F(T)}{T} .
\end{gathered}
$$

Для анализа характера ФП нами был использован гистограммный метод анализа данных, полученных методом МК $[33,34]$. Расчеты проводились для систем с 
периодическими граничными условиями и линейными размерами $L=12-120$, число частиц в системе при этом составляло $N=3 / 4 \times L \times L$.

\section{3. Результаты моделирования}

Для наблюдения за температурным ходом теплоемкости и восприимчивости использовались выражения $[35,36]$

$$
\begin{gathered}
C=\left(N K^{2}\right)\left(\left\langle U^{2}\right\rangle-\langle U\rangle^{2}\right), \\
\chi= \begin{cases}(N K)\left(\left\langle m^{2}\right\rangle-\langle|m|\rangle^{2}\right), & T<T_{N}, \\
(N K)\left\langle m^{2}\right\rangle, & T \geq T_{N},\end{cases}
\end{gathered}
$$

где $K-\left|J_{1}\right| / k_{B} T, N-$ число частиц, $U-$ внутренняя энергия, $m$ - параметр порядка $(U$ и $m$ являются нормированными величинами).

Параметр порядка системы вычислялся с помощью выражения [9]

$$
m=\frac{1}{3}\left(\left|m_{1}\right|+\left|m_{2}\right|+\left|m_{3}\right|\right),
$$

где $m_{i}$ - намагниченность в расчете на один спин подрешетки с номером $i$.

На рис. 1 приведена магнитная структура основного состояния для исследуемой модели. Черными кружками изображены спины, направленные вверх, а светлыми направленные вниз. В основном состоянии спины в одной из трех подрешеток направлены вверх, в другой подрешетке - вниз, а в третьей - или верх, или вниз. Как видно из получаемого таким образом рисунка, основное состояние имеет ферримагнитное упорядочение.

Плотность состояний $g(E)$ для систем с различными линейными размерами $L$ представлена на рис. 2 (здесь и далее статистическая погрешность не превышает размеров символов, использованных для построения зависимостей, энергия приведена в единицах $\left.\left|J_{1}\right| / k_{B}\right)$. Для удобства восприятия в виде символов приведены лишь

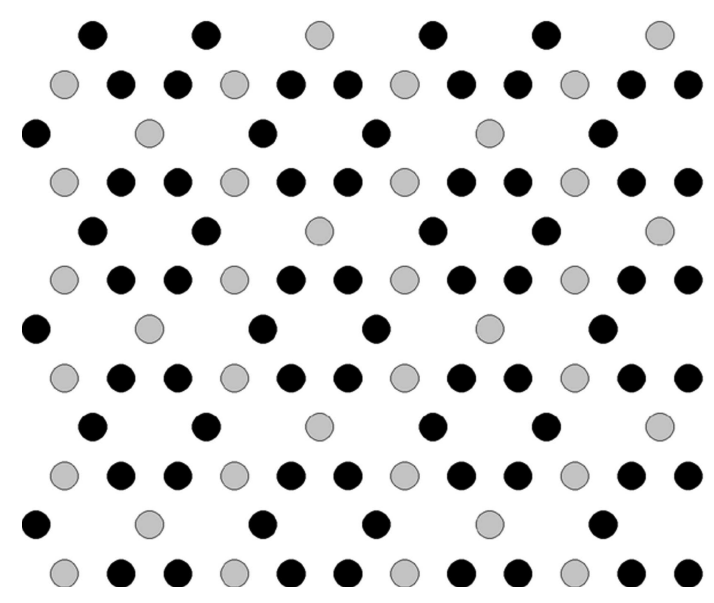

Рис. 1. Магнитная структура основного состояния.

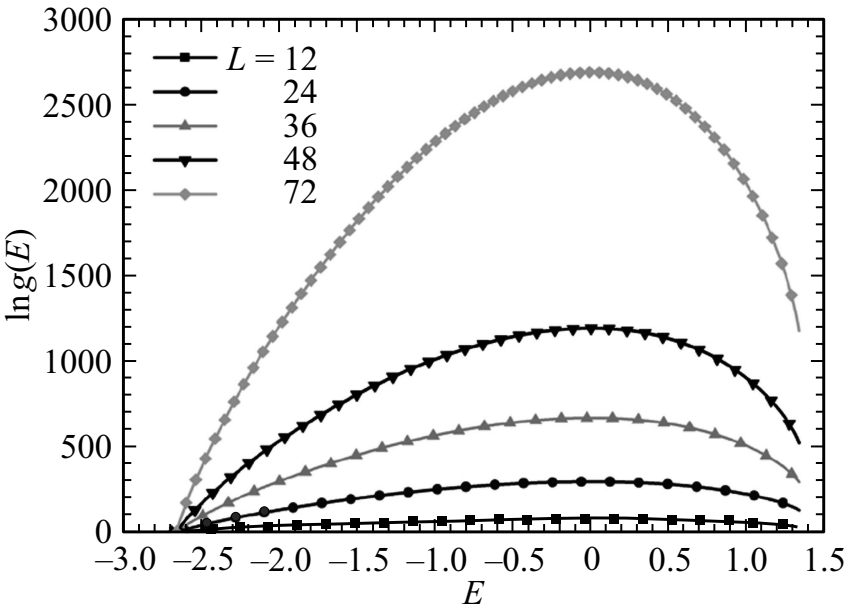

Рис. 2. Плотность состояний $g(E)$ для систем с разными линейными размерами $\mathrm{L}$.

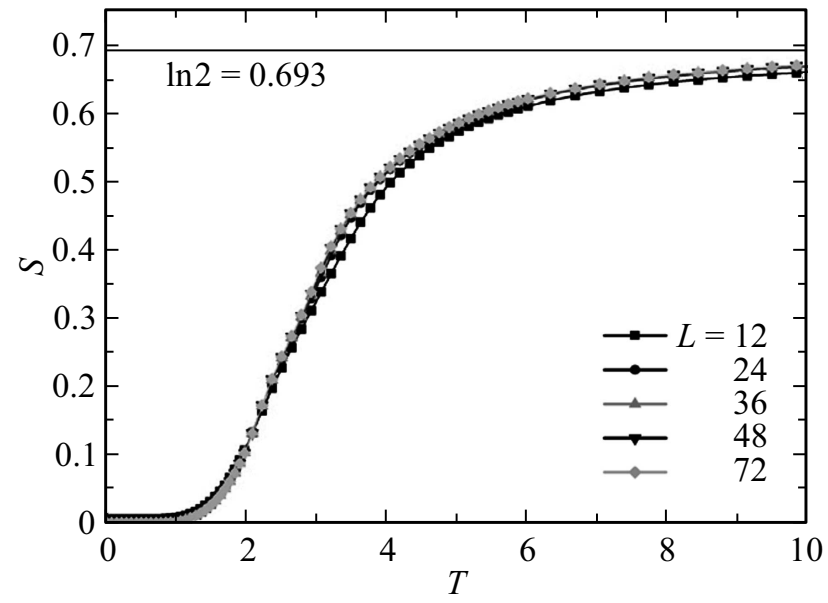

Рис. 3. Температурные зависимости энтропии $S$.

некоторые данные, линия проходит через все точки. Из графика видно, что вырождение основного состояния в данной системе отсутствует. Мы считаем, что это обусловлено тем, что в данной модели учитывается обменное взаимодействие следующих ближайших соседей, которое может способствовать частичному снятию вырождения.

Температурные зависимости энтропии $S$ при различных линейных размерах системы приведены на рис. 3 (здесь и далее температура дана в единицах $\left|J_{1}\right| / k_{B}$ ). С увеличением температуры энтропия системы стремится к теоретически предсказанному значению $\ln 2$. При низких температурах, близких к абсолютному нулю, энтропия системы стремится к нулю, в то время как для той же модели с взаимодействиями только ближайших соседей энтропия стремится к значению, отличному от нуля. Такое поведение энтропии свидетельствует о влиянии взаимодействий следующих ближайших соседей на ТС-модели. 


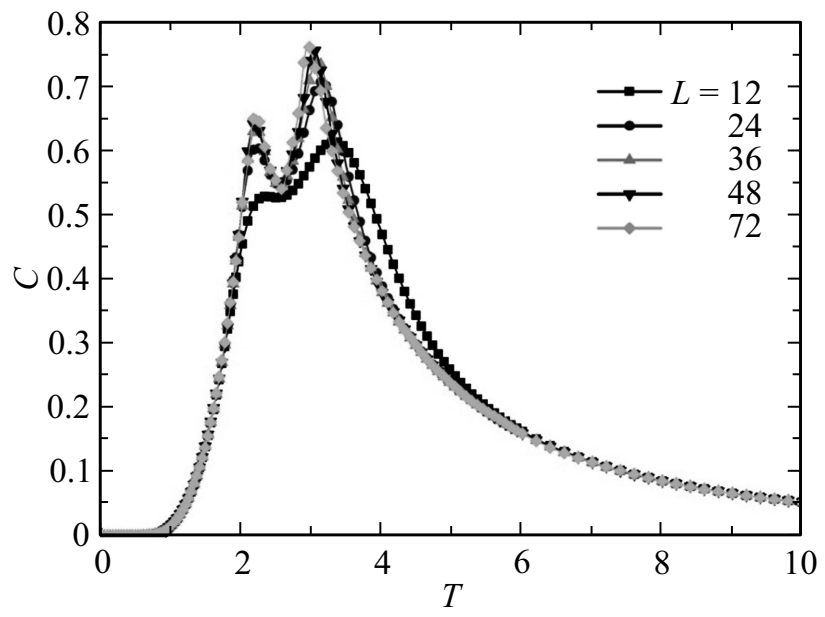

Pис. 4. Температурные зависимости теплоемкости $C$.

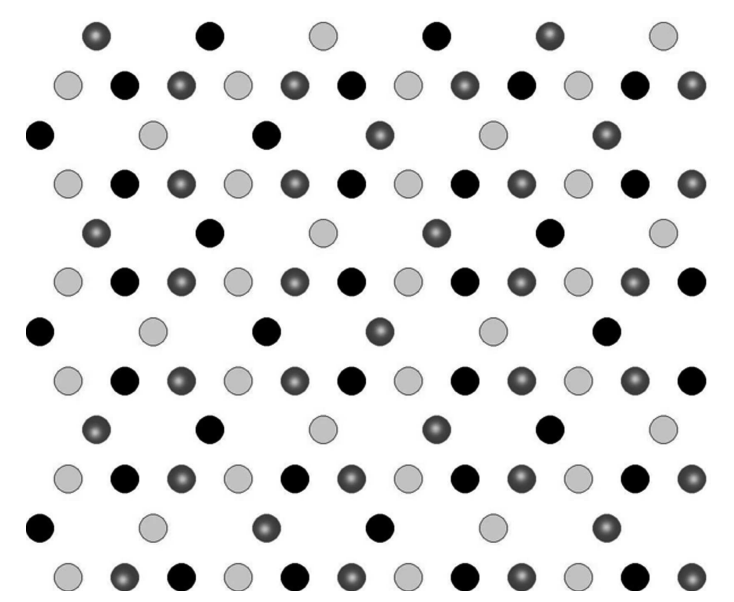

Рис. 5. Магнитная структура частично упорядоченного состояния.

На рис. 4 представлены температурные зависимости теплоемкости при различных линейных размерах системы. Отметим, что в данной модели как для теплоемкости, так и для магнитной восприимчивости наблюдается необычное поведение, которое характеризуется наличием двойного пика. На рис. 4 видно, что с увеличением линейных размеров решетки наблюдается рост абсолютных значений максимумов теплоемкости. При этом двухпиковая структура становится более отчетливой. Такое поведение, видимо, связано с конкуренцией ближайших и следующих ближайших соседей. Отметим, что первый максимум обусловлен переходом системы из упорядоченного состояния в частично упорядоченное состояние, а второй пик соответствует переходу системы из частично упорядоченного в парамагнитное состояние.

Структура частично упорядоченного состояния приведена на рис. 5. В отличие от структуры на рис. 1, в данном случае часть узлов, обозначенных на рисунке темно-серым цветом, будут иметь случайное направление спинов (или вверх, или вниз). Причем доля направ- ленных вверх и направленных вниз спинов меняется с температурой: при температурах ниже точки первого максимума теплоемкости все спины направлены вверх, а при достижении температуры второго максимума доля направленных вверх и вниз спинов выравнивается.

Температурные зависимости параметра порядка $m$ при различных линейных размерах системы приведены на рис. 6. Как видно из рисунка, наблюдается необычное поведение параметра порядка, которое становится более выраженным с ростом линейных размеров систем. Эти особенности приходятся на те же значения температур, при которых наблюдались два максимума на графике теплоемкости (рис. 4). Очевидно, что такое поведение параметра порядка также связано с переходами упорядоченное состояние-частично упорядоченное состояние-разупорядоченное состояние.

На рис. 7 приведены гистограммы распределения энергии для систем с различными линейными размерами. Графики построены в точке, соответствующей температуре второго максимума теплоемкости. В связи с тем, что температуры максимумов теплоемкости для

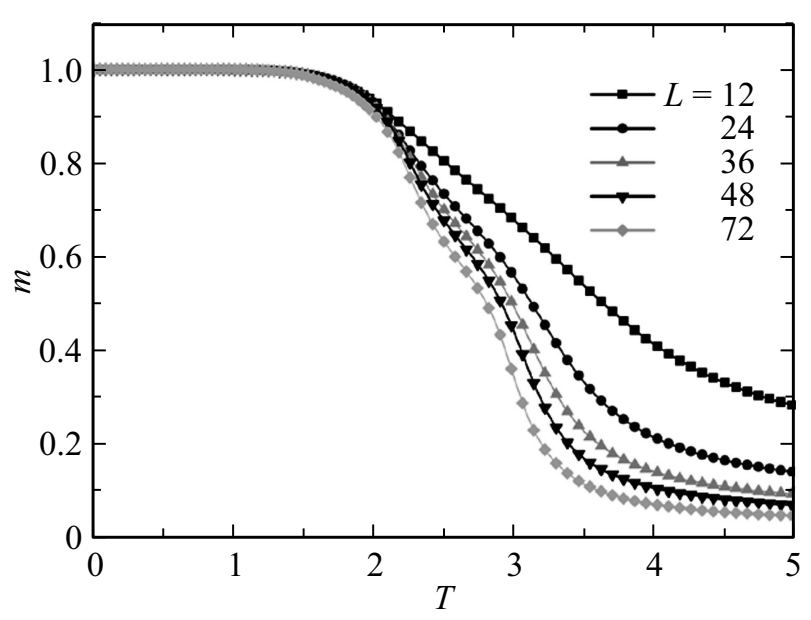

Рис. 6. Температурные зависимости параметра порядка $m$.

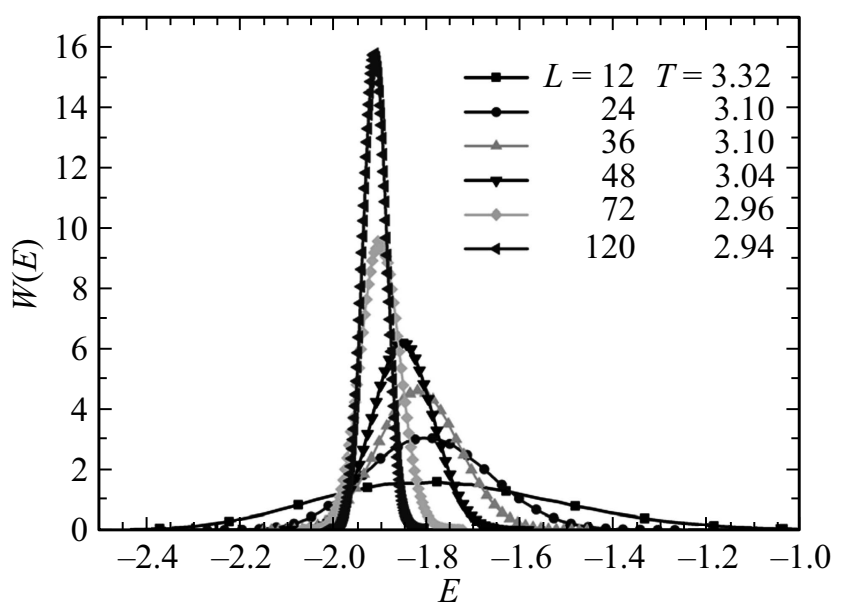

Рис. 7. Гистограммы распределения энергии $W(E)$. 
систем с разными линейными размерами отличаются, на графиках приведены соответствующие максимумам температуры. Все гистограммы нормированы таким образом, чтобы интеграл от $W(E)$ (суммарная вероятность всех энергетических состояний) равнялся единице. На графиках мы наблюдаем один пик, что характерно для ФП второго рода [33,34]. Анализируя наши данные, можно предположить, что учет ферромагнитных взаимодействий следующих ближайших соседей в двумерной антиферромагнитной модели Изинга на решетке Кагоме приводит к появлению ФП второго рода и способствует необычному поведению температурной зависимости термодинамических параметров.

\section{4. Заключение}

Исследование фазовых переходов в антиферромагнитной модели Изинга на решетке Кагоме с учетом взаимодействия вторых ближайших соседей выполнено с использованием высокоэффективного алгоритма Ванга-Ландау методом Монте-Карло. Проведен анализ структуры основного состояния и обнаружено ферримагнитное упорядочение в системе. На основе гистограммного метода проведен анализ характера фазовых переходов. Показано, что в исследуемой модели наблюдается фазовый переход второго рода. Обнаружено аномальное поведение температурной зависимости термодинамических параметров.

\section{Список литературы}

[1] P. Chandra, P. Coleman, I. Ritchey. J. de Phys. 33, 591 (1993).

[2] J.T. Chalker, P.C.W. Holdsworth, E.F. Shender. Phys. Rev. Lett. 68, 855 (1992).

[3] A.B. Harris, C. Kallin, A.J. Berlinsky. Phys. Rev. B 45, 2899 (1992).

[4] Р.С. Гехт, И.Н. Бондаренко. ЖЭТФ 113, 2209 (1998).

[5] А.К. Муртазаев, М.К. Рамазанов, М.К. Бадиев. ФНТ 37, 1258 (2011).

[6] F.A. Kassan-Ogly, B.N. Filippov, A.K. Murtazaev, M.K. Razanov, M.K. Badiev. JMMM 324, 3418 (2012).

[7] А.К. Муртазаев, М.К. Рамазанов, Ф.А. Кассан-Оглы, М.К. Бадиев. ЖЭТФ 144, 1239 (2013).

[8] А.Б. Бабаев, М.А. Магомедов, А.К. Муртазаев, Ф.А. Кассан-Оглы, А.И. Прошкин. ЖЭТФ 149, 357 (2016).

[9] M. Wolf, K.D. Schotte. J. Phys. A: Math. Gen. 21, 2195 (1988).

[10] R.S. Gekht, V.I. Ponomarev. Phase Transitions 20, 27 (1990).

[11] D.P. Landau, K. Binder. Monte Carlo Simulations in Statistical Physics. Cambridge University Press, Cambridge (2000).

[12] T. Matsuo, H. Suga. Rev. Inorg. Chem. 3, 371 (1981).

[13] M. Stahn, R.E. Lechner, H. Dachs, H.E. Yacobs. J. Phys. C: 16, 5073 (1983).

[14] R. Wang, W.F. Bradley, H. Steinfink. Acta Crystallogr. 18, 249 (1965).

[15] Y.L. Loh, D.X. Yao, E.W. Carlson. Phys. Rev. B 77, 134402 (2008).
[16] M.G. Townsend, G. Longworth, E. Roudaut. Phys. Rev. B 33, 4919 (1986).

[17] M. Takano, T. Shinjo, T. Takada. J. Phys. Soc. Jpn. 30, 1049 (1971).

[18] T. Takagi, M. Mekata. J. Phys. Soc. Jpn. 62, 3943 (1993).

[19] А.К. Муртазаев, И.К. Камилов, М.А. Магомедов. ЖЭТФ 120, 1535 (2001).

[20] A.K. Murtazaev, I.K. Kamilov, M.A. Magomedov, Comp. Phys. Commun. 147/1-2, 447 (2002).

[21] А.К. Муртазаев, М.К. Рамазанов. ФТТ 53, 1004 (2011).

[22] А.К. Муртазаев, М.К. Рамазанов, М.К. Бадиев. ЖЭТФ 142, 338 (2012).

[23] М.А. Магомедов, А.К. Муртазаев, Л.К. Магомедова. Вестн. ДГУ 31, 71 (2016).

[24] A.K. Murtazaev, A.B. Babaev, M.A. Magomedov, F.A. Kassan-Ogly, A.I. Proshkin. Solid State Commun. 246, 41 (2016).

[25] М.А. Магомедов, А.К. Муртазаев, Л.К. Магомедова. Вестник ДГУ 31, 43 (2016).

[26] M.K. Ramazanov, A.K. Murtazaev, M.A. Magomedov. Solid State Commun. 233, 35 (2016).

[27] F. Wang, D.P. Landau. Phys. Rev. E 64, 056101 (2001).

[28] D.P. Landau, S.-H. Tsai, M. Exler. Am. J. Phys. 72, 1294 (2004).

[29] A.G. Cunha-Netto, A.A. Caparica, S.H. Tsai, R. Dickman, D.P. Landau. Phys. Rev. E 78, 55701 (2008).

[30] A. Bunker, B. Gaulin, C. Kallin, Phys. Rev. B 48, 15861 (1993).

[31] C. Zhou, R.N. Bhatt. Phys. Rev. E 72, 025701 (2005).

[32] X. Yao. Phys. Lett. A 377, 342 (2013).

[33] F. Wang, D.P. Landau, Phys. Rev. Lett. 86, 2050 (2001).

[34] М.К. Рамазанов. Письма в ЖЭТФ 94, 335 (2011).

[35] K. Binder, J.-Sh. Wang. J. Status Phys. 55, 87 (1989).

[36] P. Peczak, A.M. Ferrenberg, D.P. Landau. Phys. Rev. B 43, 6087 (1991). 